Acta Crystallographica Section D

Biological

Crystallography

ISSN 0907-4449

\section{Gwyndaf Evans, * Pietro Roversi and Gérard Bricogne}

MRC Laboratory of Molecular Biology, Hills Road, Cambridge CB2 2QH, England

Correspondence e-mail: gwyndaf@mrc-Imb.cam.ac.uk

\title{
In-house low-resolution X-ray crystallography
}

It is demonstrated that standard in-house protein crystal X-ray diffraction apparatus can be used to measure very low resolution reflections with only a few modifications. The apparatus and modifications are described in detail and tested on two different macromolecular crystal samples: lysozyme and the $30 \mathrm{~S}$ ribosomal subunit. Contrast-variation measurements on tetragonal hen egg-white lysozyme demonstrate the potential usefulness of the apparatus in providing accurate data for the determination of macromolecular envelopes. In contrast, the measurement of very low resolution diffraction from crystals of the $30 \mathrm{~S}$ ribosome subunit illustrates how inhouse facilities can provide data from small weakly diffracting crystals of a very large macromolecule.

\section{Introduction}

A common property shared by all protein crystals is their biphasic nature (Bernal \& Crowfoot, 1934). That is, each unit cell consists of one or more ordered protein molecules surrounded by a highly disordered solvent region. The solvent typically consists of water, buffer and salt molecules and sometimes cryo-protectant molecules. The boundary between protein and solvent is typically fairly well defined and usually extends across a hydration layer only a few ångströms deep (Svergun et al., 1998). This boundary may be represented in terms of a surface or envelope which describes the threedimensional shape of a protein molecule. Inside this envelope, atoms are assumed to be ordered and to contribute to diffraction intensities at high resolution. Outside the envelope, atoms are disordered and difficult to model as a large number of single atoms with individual positional and thermal displacement parameters. This region is, therefore, normally assumed to have a constant electron density and a high temperature factor and thus to contribute only to lowresolution diffraction intensities.

Research into the determination of the protein envelope in a crystal using X-ray diffraction started with the work of Bragg \& Perutz (1952). A few years earlier, Boyes-Watson et al. (1947) had already observed that substitution of ammonium sulfate for water as the solvent in crystals of horse methaemoglobin lowered the intensities of low-order reflections but left higher order intensities unchanged. These changes were interpreted as being a consequence of variation of the mean electron density of the solvent surrounding the protein molecules under the assumption that the protein molecules themselves were being left unchanged. By making diffraction intensity measurements under five different conditions corresponding to a salt-free solvent and four different salt concentrations, Bragg \& Perutz (1952) were able
Received 24 February 2000 Accepted 22 June 2000
(C) 2000 International Union of Crystallography Printed in Denmark - all rights reserved 
to deduce an approximate form and size for the haemoglobin molecule.

This was the first example of the X-ray contrast-variation technique for the determination of protein envelopes. Since then, efforts have been made to determine more detailed envelopes using solvent-contrast effects (Carter et al., 1990; Carter, 1998), the related technique of multiwavelength anomalous solvent contrast (MASC; Ramin et al., 1999; Fourme et al., 1995) and direct phasing approaches (Lunin et al., 1998; Subbiah, 1991). These methods attempt to extract the information describing the form of the protein envelope from diffraction intensities at low resolution $\left(d_{\mathbf{h}}>10 \AA\right)$. Apart from a small number of exceptions, the measurement of these low-resolution data has been neglected for many years. The reason for this is partly that the effects of bulk solvent on lowresolution data (Phillips, 1980) has been neglected in crystallographic software until recently (Jiang \& Brünger, 1994) and partly because in the everyday job of determining protein crystal structures by well established methods such as MIR(AS), SIR(AS), MR and MAD ${ }^{\mathbf{1}}$ low-resolution data are not always thought to be essential. Typical protein diffraction data sets will extend only as low as $20 \AA$ resolution and in the worst cases these data may be saturated on the detector or corrupted by the presence of a poorly positioned or poorly designed beamstop. The lowest order reflections are almost never measured and thus are rarely exploited in terms of their usefulness in describing the molecular envelope.

A notable exception is the work of Harrison \& Jack (1975), where signs and phases from small-angle $\mathrm{X}$-ray scattering and very low resolution X-ray crystal diffraction (Harrison, 1969) and a $28 \AA$ electron microscopy (EM) reconstruction (Jack et al. 1975) were used to determine the positions of clusters of $\mathrm{PtCl}_{6}^{2-}$ ions in crystals of tomato bushy stunt virus. Indeed, the combination of EM and X-ray crystallography is a recurring theme in both virus work (Prasad et al., 1999; Wynne et al., 1999; Dokland et al., 1998; Simpson et al., 1998; Grimes et al., 1997; Rayment et al., 1982) and in the determination of very large macromolecule structures (Harms et al., 1999; Ban et al., 1998; Glykos et al., 1998; Wang et al., 1997). In most cases, EM phases are used either to initiate or corroborate crystal structure solution. However, problems arise in the combination of EM and X-ray observations, since there is typically only a small range of resolution over which the two sources of information overlap. EM reconstructions are only usually informative to resolutions where $d_{\mathbf{h}}>15 \AA$ with very few exceptions, the most notable being the $7.4 \AA$ reconstruction of the core protein of hepatitis B virus (Böttcher et al., 1997). $\mathrm{X}$-ray data, on the other hand, rarely extend into the lowresolution region. There exists, therefore, an unfulfilled need for the routine measurement of very low resolution diffraction data from macromolecular crystals as a first step towards extending the region of overlap between the EM and X-ray data.

\footnotetext{
${ }^{1}$ Abbreviations used: MIR(AS), multiple isomorphous replacement (with anomalous scattering); SIR(AS), single isomorphous replacement (with anomalous scattering; MR, molecular replacement; MAD, multiple wavelength anomalous diffraction.
}

The measurement of very low resolution data is usually associated with the use of synchroton radiation because of the good intrinsic collimation of the X-rays. Indeed, specialist facilities for the measurement of low-resolution X-ray crystal diffraction data are now being established (Tsuruta et al., 1998). However, there still exists a preference by many experimenters towards measuring data in the home laboratory, as access to synchrotrons can still be restricted and involve delays of many months. Very low order reflections typically produce the strongest intensities, sometimes orders of magnitude stronger that the average intensities of reflections measured between 10 and $2 \AA$. This makes the very low order reflections accessible to measurement using conventional sources, even from relatively weakly diffracting crystals. Miller et al. (1999) have measured very low resolution reflections from poliovirus crystals using a rotating anode and a MAR 345 detection system. They collimated the X-ray beam using a 'pinhole' to produce a fine X-ray beam with an inevitable reduction in the X-ray flux incident on the sample. With this configuration, the lowest order reflections were measurable using exposure times as low as $15 \mathrm{~s}$. Indeed, some of the earliest experiments referred to above were performed in-house on conventional sources and best demonstrate the possibilities if care is taken with instrumental design and setup (Harrison, 1968; Perutz \& Rogers, 1946).

In this paper, we present a modified X-ray diffraction camera which allows the accurate measurement of very low resolution reflections corresponding to Bragg spacings of $200 \AA ̊$ or greater using a conventional rotating-anode source and a commercial image-plate system while avoiding any significant loss in X-ray flux.

\section{Experimental apparatus}

\subsection{Requirements of the experiment}

Setting up a low-angle X-ray camera requires the removal of as much low-angle scattered radiation as possible, the aim being to minimize the level of X-ray background recorded on an area detector and ideally to make the background smoothly varying. Possible sources of unwanted background arise from (i) scatter caused by the interaction of the main X-ray beam with air before and after the specimen, (ii) scatter from collimating slits, (iii) scatter from filters and windows and (iv) scatter from disordered regions of the specimen itself including bulk solvent, the accompanying solvent surrounding the crystal and the loop material. We consider here only sources of scatter from the apparatus itself and will neglect the specimen as a source of contaminating scattering.

A fundamental principle in the design of low-angle X-ray cameras is the positioning of collimating slits sufficiently far upstream of the sample so that the scatter produced by those slits is sufficently well separated from the main beam path so as to allow its removal by a guard aperture positioned just before the sample (Huxley, 1953; Bolduan \& Bear, 1949). We have attempted to use this idea and a helium-filled path to 
reduce the above-mentioned sources of background as much as possible.

Most crystal diffraction cameras use a beamstop mounted as close to the crystal as possible so that air scatter is reduced without obscuring too much low-resolution data. Even so, data below $15 \AA$ are typically missing or corrupted as a result of the finite size of the sample crystal. Reflections recorded on the detector surface within the penumbra of the beamstop shadow are systematically wrong because they are a result of diffraction from only a fraction of the total diffracting volume of the sample (see Fig. 3 in Carter, 1998). For this reason, the use of a beamstop position as close to the detector as possible is favoured. This in turn, however, forces the use of an evacuated beam path or alternatively a helium-filled air path between sample and detector.

\subsection{Realisation of the requirements}

The X-ray source used in this work was an Elliot GX-13 rotating anode with a foreshortened X-ray source size of $100 \times 100 \mu \mathrm{m}$. Fig. 1 is a schematic diagram illustrating the diffraction camera downstream of the X-ray source. The generated X-rays are focused horizontally and vertically by $60 \mathrm{~mm}$ bent mirrors (Charles Supper Company, Natick, MA, USA). The mirror pair is immediately followed by a set of vertical and horizontal slits (SO) and then a $300 \mathrm{~mm}$ MAR imaging plate and standard base. The MAR system consists of a collimator assembly, sample-rotation axis, beamstop mount and translatable detector all mounted on an adjustable base allowing alignment into the X-ray beam. The collimator assembly houses two sets of vertical and horizontal slits positioned $110 \mathrm{~mm}$ ( $S 1$ in Fig. 1) and $60 \mathrm{~mm}$ ( $S 2$ in Fig. 1) upstream of the sample position. Each vertical and horizontal slit pair is followed by an ionization chamber which helps in base alignment. Just upstream of the sample is a removable collimator nose-piece designed to remove scatter introduced

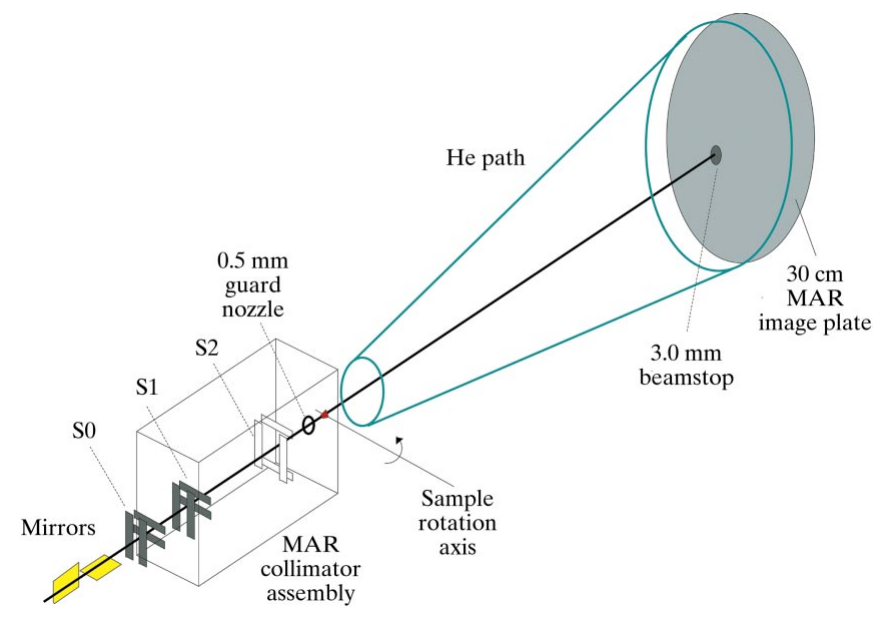

Figure 1

Schematic overview of the diffraction camera used for the low-resolution measurements. From left to right are the Supper mirrors (Charles Supper) and mirror exit slits, $S 0$, the MAR collimator front set of slits, $S 1$, a modified collimator nose-piece or guard nozzle, the sample position, helium flight tube, beamstop and image-plate detector. by the slits and air path within the collimator assembly. Nosepiece aperture diameters of 1.0, 1.5 and $2.0 \mathrm{~mm}$ are available with the detector; however, for this work a set of smaller diameter apertures were used, the smallest having a diameter of $0.5 \mathrm{~mm}$. Alignment of the small aperture nose pieces with respect to the collimator slits was performed using an ionization chamber to monitor the flux exiting the collimator with and without the nose-piece in place. $S 1$ and $S 2$ slit openings of $0.25 \mathrm{~mm}$ vertically and horizontally were used during alignment, although during the measurement of low-resolution data slit $S 2$ was opened fully and essentially excluded from the experiment.

The helium-filled flight path was constructed out of a transparent plastic bag which had been cut to shape and then heat sealed to form a cone. This bag was held at both ends by two flanges, one of $300 \mathrm{~mm}$ diameter at the detector side and one of $150 \mathrm{~mm}$ diameter near the sample. A small plastic funnel was cut to form a cone and then mounted onto the smaller flange to taper the helium path down to form an entrance window aperture of $15 \mathrm{~mm}$ in diameter. A $3 \mu \mathrm{m}$ thick mylar window was placed over the entrance aperture and held in place with vacuum grease. The exit window was a $20 \mu \mathrm{m}$ mylar film and had a $3 \mathrm{~mm}$ diameter beamstop glued to its centre on the inside surface. The beamstop was made by turning out a $3.0 \mathrm{~mm}$ diameter and $5 \mathrm{~mm}$ long aluminium rod and filling it with lead-tin solder. It was fixed to the He path window using Loctite Superglue. Adjustment of the path and beamstop position was made manually by loosening set screws which fixed the path to the MAR detector base and recording positions relative to the base using callipers. The front window of the path was placed as close to the sample position as possible without interfering with the nitrogen and dry air gas stream cooling the crystal. In the early stages of flooding the path with helium, the front mylar window acted as a moisture trap owing to its proximity to the Cryostream and some ice formation on the inside of the window was observed. Once all moisture in the path had been replaced, however, this problem no longer arose.

Fig. 2 shows the positions of each element of the diffraction camera relative to the source. The $100 \mathrm{~mm}$ space between the last defining slit and the nose-piece just before crystal is essential for producing a clean X-ray beam free of contaminating scatter. Fig. 2 illustrates this point. After being focused by the mirrors and collimated by slits $S 0$ and $S 1$, the main $\mathrm{X}$-ray beam passes uninterrupted through the nose-piece onto the sample and then through the He path until it is absorbed by the beamstop. The size and alignment of the nose-piece is critical, since any interaction of the X-ray beam with the nosepiece would cause significant scatter into the low-resolution $\mathrm{X}$-ray pattern. The aperture diameter must be large enough to allow the main beam through and small enough to confine any scatter produced by $S 1$ to the beamstop. The sizes of the slits $S 0$ and $S 1$ were 0.4 and $0.35 \mathrm{~mm}$, respectively, in both horizontal and vertical directions. The $\mathrm{X}$-ray flux measured at the sample was comparable with the flux measured during normal operation of the camera using similar settings for both pairs of slits in the MAR collimator assembly. The relatively poor 
calibration of slits in this setup meant that the process of producing a clean X-ray camera free of excess background and slit scatter was an iterative process relying on observation as well as careful adjustment of apertures. Consequently, alignment can take as much as a day, as one relies completely on viewing detector images of the camera scatter. Better calibrated apertures would speed up this process by improving control over the experimental geometry.

\section{Test case I: tetragonal hen egg-white lysozyme}

To investigate the X-ray camera's suitability for performing contrast-variation experiments, we conducted experiments using the tetragonal form of hen egg-white lysozyme (HEWL). HEWL acts as a sufficiently robust and reproducible sample with which to perform test studies. It crystallizes readily even in the presence of cryo-protectant and its size and morphology can be controlled (Forsythe et al., 1999) to produce test samples which emulate conditions potentially experienced in 'real life'.

\subsection{Sample preparation}

The crystals used in this study were grown from $1.5 \mathrm{M} \mathrm{NaCl}$, $25 \%$ ethylene glycol and $0.1 M$ sodium acetate $\mathrm{pH}$ 4.7. In order to measure solvent-contrast effects, the crystals were soaked just prior to freezing in the Oxford Cryosystems Cryostream in two solutions (hereafter referred to as low and high density) having different electron densities. Although the crystals had been grown in the presence of a relatively low electron density solution, it was necessary to re-soak for $30 \mathrm{~s}$ in a better defined low-density solution so that an accurate estimate of the mean solvent electron density, $\rho_{s}$, in the crystal could be made. The low-density solution was $2 M \mathrm{NaCl}, 25 \%$

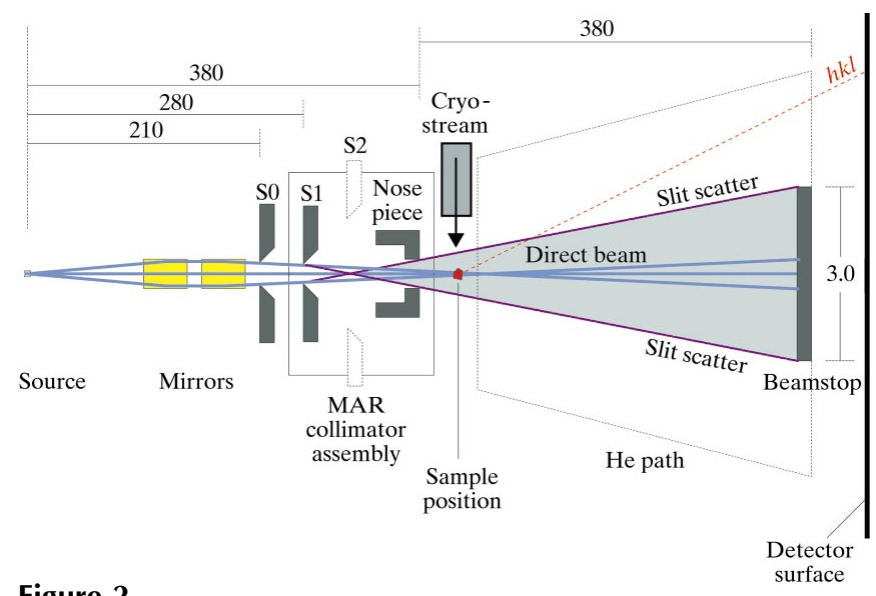

Figure 2

Illustration of the geometry of the low-resolution diffraction camera. Dimensions are in $\mathrm{mm}$. (For clarity, the scale is expanded in the vertical direction by a factor of about 70 relative to the horizontal.) The collimating slits $S 0$ and $S 1$ act as a source of unwanted scattered radiation which can appear as linear streaks extending into the diffraction pattern outwards from the beam centre. This scatter is confined to lie inside the area obscured by the beamstop (shaded area) by the use of a guard aperture, in this case a modified collimator nose piece. Low-resolution diffracted X-rays (shown in red labelled $h k l$ ) pass through the He path and are incident on the detector surface. ethylene glycol and $0.1 M$ sodium acetate at $\mathrm{pH} 4.7$ having $\rho_{s}=0.349 \mathrm{e}^{-3}$. A higher salt concentration was used in this case to stabilize the crystals in the absence of protein in solution.

The high-density crystals were obtained by soaking in a solution containing $2 M \mathrm{NaCl}, 2 M$ D-glucose and $0.1 M$

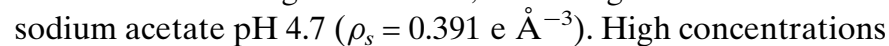
of D-glucose have previously been used by Yu \& Caspar (1998) in the measurement of cubic insulin diffraction data. As well as increasing the mean electron density of the solvent, the glucose also acted as a cryo-protectant.

The mean electron densities of the soaking solutions were determined by careful volume and weight measurements of

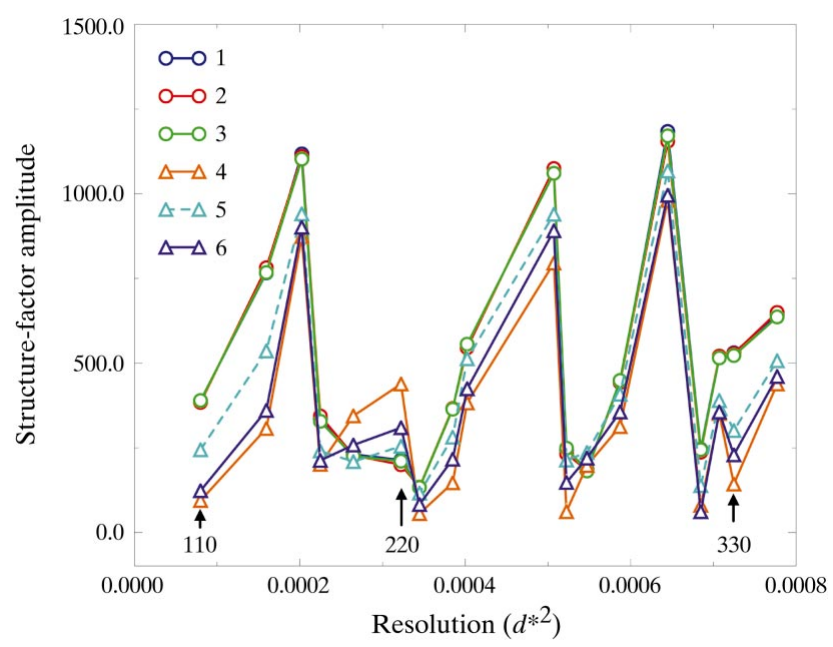

Figure 3

Structure-factor amplitudes for HEW lysozyme's 18 lowest resolution reflections $(56-35 \AA$ ) plotted for each of the six data sets. The diagram illutrates the high level of consistency between the three low-density data sets ( 1 to 3 ) even at the level of a single observation. Good consistency is seen also between data sets 4 and 6 with set 5 (dashed) appearing as an outlier at very low resolution.

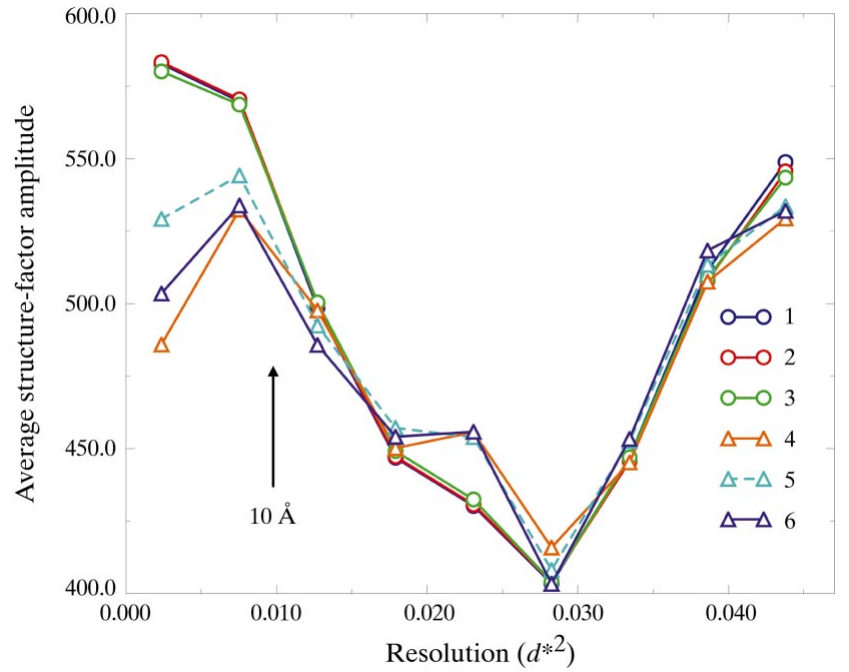

Figure 4

The binned average structure-factor amplitudes for all data (56-4.59 $\mathrm{A})$ plotted for the six HEWL data sets. The potential outlier data set 5 is shown dashed. 
Table 1

HEWL crystal and data-collection parameters and the results of data analysis using MOSFLM and SCALA.

Crystal and data-collection parameters.

\begin{tabular}{ll}
\hline Space group & $P 4_{3} 2_{1} 2$ \\
Resolution $(\AA)$ & $56.0-4.59$ \\
Exposure time $(\min )$ & $3-20$ \\
Oscillation angle $\left(^{\circ}\right)$ & $3(4$ for crystal 5) \\
Total rotation range $\left(^{\circ}\right)$ & $200-360$ \\
Solvent electron density $\rho_{s}\left(\AA^{-3}\right)$ & $0.349($ crystal 1,2 and 3) \\
Solvent electron density $\rho_{s}\left(\AA^{-3}\right)$ & 0.391 (crystal 4,5 and 6) \\
\hline
\end{tabular}

Summary of results.

\begin{tabular}{lllllll}
\hline Crystal & $\begin{array}{l}a=b \\
(\AA)\end{array}$ & $\begin{array}{l}c \\
(\AA)\end{array}$ & $\begin{array}{l}\text { Mosaicity } \\
\left.{ }^{\circ}\right)\end{array}$ & Multiplicity & $\begin{array}{l}\text { Completeness } \\
(\%)\end{array}$ & $R_{\text {merge }}$ \\
\hline 1 & 78.81 & 36.80 & 0.2 & 19.1 & 100 & 3.4 \\
2 & 78.76 & 36.79 & 0.2 & 13.2 & 100 & 4.3 \\
3 & 78.78 & 36.84 & 0.2 & 24.3 & 100 & 3.1 \\
4 & 78.11 & 37.51 & 0.3 & 23.9 & 100 & 4.4 \\
5 & 78.12 & 37.10 & 0.9 & 13.1 & 100 & 5.3 \\
6 & 77.72 & 37.24 & 0.3 & 16.2 & 100 & 3.4 \\
\hline
\end{tabular}

the substances used in preparing the solution. This included making a record of the total amount of water added to the solutions to make up final volumes. It is estimated that the values of $\rho_{s}$ given above are measured to better than $0.5 \%$. It is highly unlikely that better accuracy is necessary given that the bulk-solvent density may differ from that of the prepared solutions because of protein boundary effects and/or incomplete penetration of the solution into the crystal.

The soaking times used for the high-density crystals were of the order of 1-2 min. The length of soak was based on observation of the crystal integrity. The crystals were not particularly stable in the high-density solution and in some cases crystals cracked owing to osmotic shock.

\subsection{Measurement of diffraction data}

The lowest resolution reflection from this tetragonal form of lysozyme appears at a resolution of about $56 \AA$. At a sampleto-detector distance of $425 \mathrm{~mm}$ the edge of the detector corresponded to a high-resolution limit of $4.58 \AA$. The beam path was flooded with helium and a $0.58 \mathrm{~mm}$ diameter nosepiece was inserted into the collimator end. At this stage some scatter was observed at resolutions with $d_{\mathbf{h}}>100 \AA$ but this was acceptable as it did not interfere with the low-resolution reflections or surrounding background. Data were recorded from a total of six crystals: three at low density and three at high density. The crystals were frozen directly into a nitrogen Cryostream operating at $100 \mathrm{~K}$. The data-collection parameters and results of analysis with MOSFLM (Leslie, 1992) and SCALA (Collaborative Computational Project, Number $4,1994)$ are shown in Table 1. In all cases an oscillation range of $3^{\circ}$ was used, except for crystal 5 where $4^{\circ}$ oscillations were used. The total rotation range used varied but in each case all the data between 56 and $4.59 \AA$ were measured with average multiplicities greater than 13 .
A consistent decrease of $0.7-1.1 \AA$ in the $a$ and $b$ axes was observed for the high-density crystals relative to the lowdensity crystals. Similarly, the $c$ axis was found to increase by $0.3-0.7 \AA$. The mosaicities of the low-density crystals were estimated to be about $0.2^{\circ}$. The high-density crystals had consistently higher mosaicity but crystal 5 appeared to be an outlier among the six crystals with an estimated mosaicity of $0.9^{\circ}$. The higher than average $R_{\text {merge }}$ (see Table 1 ) also indicates that the crystal may not have been of as high a quality as the other five.

\subsection{Scaling}

The six data sets were placed on a common scale using SCALEIT (Collaborative Computational Project, Number 4, 1994). The scaling was performed using data between 10 and $4.59 \AA$ under the assumption that the expected contrastvariation effects are negligible for resolutions higher that $10 \AA$ and that the non-isomorphism was not significant at $4.59 \AA$. This strategy has been verified using synthetic data generated for tetragonal HEWL with four different mean solvent electron densities (unpublished results). However, no attempt has been made to model the effects of non-isomorphism on the scaling procedure. At low resolution, changes in structurefactor amplitudes owing to non-isomorphism can be expected to be small relative to the changes observed owing to contrast variation. However, the reliance upon high-resolution data for scaling imposes requirements on the level of acceptable nonisomorphism. This is evidently where the advantage of the MASC technique lies.

A further complication in contrast variation relates to the handling of the significant changes in sample absorption one can expect when the mean electron density of the solvent in and around the crystal sample is changed significantly. In the present study this difficulty has been overcome by relying upon high multiplicity of measurement and spherical harmonic secondary beam-scaling corrections implemented by Phil Evans in SCALA. Low values of $R_{\text {merge }}$ over all resolution ranges are indicative of the success of this procedure.

\subsection{Observation of contrast variation}

Figs. 3 and 4 show the effect on the low-resolution data of varying the solvent electron density. In Fig. 3 structure-factor amplitudes are plotted for data between 56 and $15 \AA$. The low contrast data sets agree to within a few percent demonstrating that data from different (but isomorphous) crystals can be recorded accurately and scaled together using only data between 10 and $4.59 \AA$, while maintaining the correct relative scale at low resolution. The high-density data sets (4-6) are less well behaved, however, with data set 5 emerging as an outlier among the three data sets. The effects of the change in solvent electron density are clearly visible in Fig. 4. Below about $10 \AA$ the magnitudes of the average structure factors between low- and high-density data sets diverge to the point where they differ by about $20 \%$. Apart from a glitch at $6.6 \AA$, the agreement between the average structure factors for all data sets at higher resolutions is within $4 \%$. 
Variation in the observed structure factors between the three high-density crystals could also be a consequence of small variations in the soaking times of the three crystals. No systematic behaviour was observed as a result; however, the effect of soaking time will form part of further studies into contrast-variation effects.

The effect of the contrast variation can be observed directly in the diffraction patterns from the low- and high-density crystals of HEWL. Fig. 5 shows regions of two $12^{\circ}$ overlay images from crystal 1 (low density) and crystal 6 (high density). In both cases, the 110, 220 and 330 reflections are

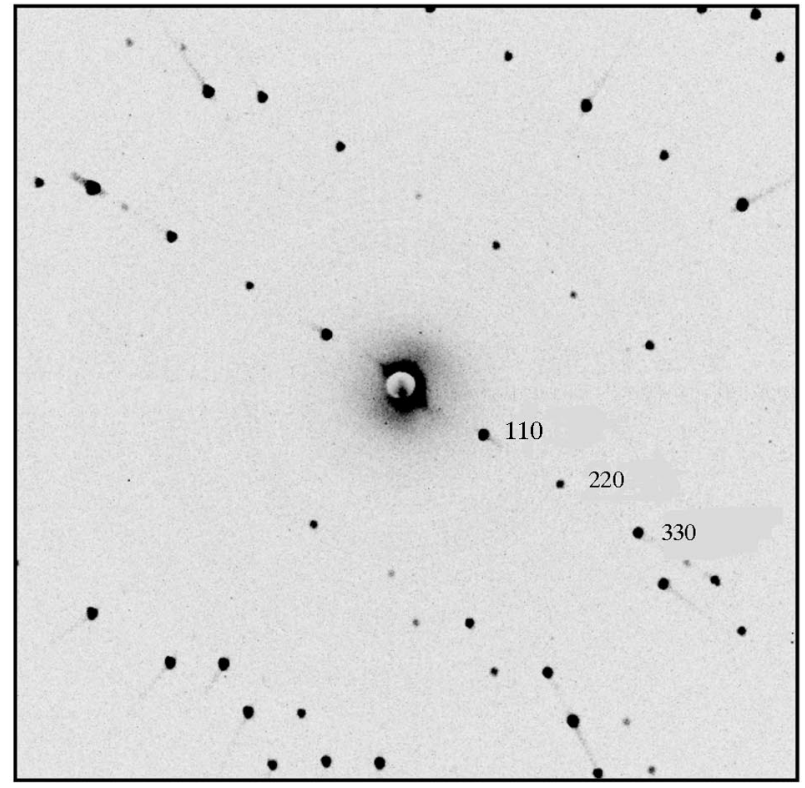

(a)

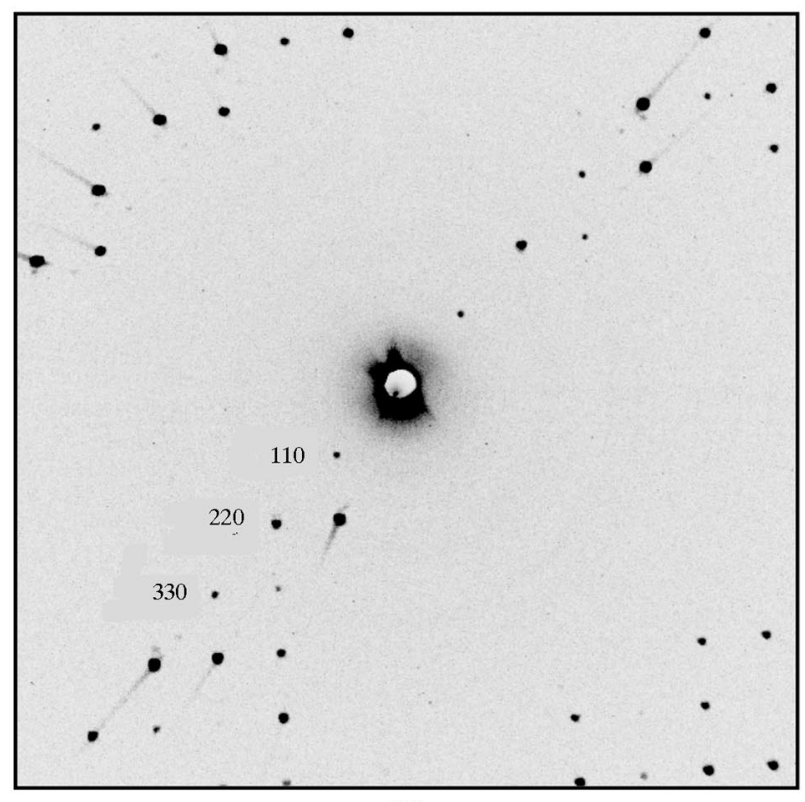

(b)

Figure 5

Two $12^{\circ}$ overlay images showing the region of reciprocal space from tetragonal HEWL crystals containing the 110, 220 and 330 reflections from $(a)$ crystal 1 having $\rho_{s}=0.349$ and $(b)$ crystal 6 having $\rho_{s}=0.391$.
Table 2

$30 \mathrm{~S}$ ribosomal subunit crystal and data-collection parameters and the results of data analysis using MOSFLM and SCALA.

Crystal and data-collection parameters.

\begin{tabular}{ll}
\hline Space group & $P 4_{1} 2_{1} 2$ \\
Unit-cell parameters $(\AA)$ & $401.5 \times 401.5 \times 174.5$ \\
Sample dimensions $(\mu \mathrm{m})$ & $200 \times 80 \times 80$ \\
Resolution $(\AA)$ & $180-18$ \\
Exposure time $(\min )$ & 10 \\
Oscillation angle $\left(^{\circ}\right)$ & 1 \\
Total rotation range $\left(^{\circ}\right)$ & 145 \\
\hline
\end{tabular}

Summary of results.

\begin{tabular}{llcccc}
\hline $\begin{array}{l}\text { Resolution } \\
(\AA)\end{array}$ & $\begin{array}{l}\text { No. } \\
\text { measured }\end{array}$ & $\begin{array}{l}\text { No. } \\
\text { unique }\end{array}$ & Multiplicity & $\begin{array}{l}\text { Completeness } \\
(\%)\end{array}$ & $R_{\text {merge }}$ \\
\hline 56.92 & 439 & 61 & 7.2 & 93.8 & 0.027 \\
40.25 & 896 & 99 & 9.1 & 100.0 & 0.029 \\
32.86 & 1170 & 123 & 9.5 & 100.0 & 0.041 \\
28.46 & 1359 & 134 & 10.1 & 100.0 & 0.053 \\
25.46 & 1569 & 153 & 10.3 & 100.0 & 0.073 \\
23.24 & 1770 & 173 & 10.2 & 100.0 & 0.091 \\
21.51 & 1790 & 173 & 10.3 & 100.0 & 0.126 \\
20.12 & 1996 & 189 & 10.6 & 100.0 & 0.188 \\
18.97 & 2073 & 200 & 10.4 & 100.0 & 0.260 \\
18.00 & 2214 & 210 & 10.5 & 100.0 & 0.299 \\
& & & & & \\
Total & 15275 & 1513 & 10.1 & 99.7 & 0.057 \\
\hline
\end{tabular}

visible and are labelled. Although the images are not on the same relative scale, one can still clearly observe changes in the intensities of the 110, 220 and 330 reflections which are, respectively, large, small and large in the low-density crystal, and small, large and small in the high-density crystal. This corresponds to the intensity changes observed and labelled in Fig. 3.

\section{Test case II: $30 \mathrm{~S}$ ribosome}

The structure of a bacterial $30 \mathrm{~S}$ ribosomal subunit was solved to $5.5 \AA$ resolution by Clemons et al. (1999). It crystallizes in the tetragonal space group $P 4_{1} 2{ }_{1} 2$, with approximate unit-cell parameters $a=b=401.5, c=174.5 \AA$. The crystal used for this study was approximately $200 \times 80 \times 80 \mu \mathrm{m}$ in size and was frozen to $100 \mathrm{~K}$. To reduce further the scatter in the very low resolution part of the diffraction pattern the MAR collimator assembly was also flooded with helium. This produced a clean diffraction camera down to about $250 \AA$.

Data-collection parameters and results of analysis are given in Table 2. The data are complete and highly redundant, apart from four reflections in the lowest resolution bin which were not present in the final merged data set. The high quality of the results suggests that the data were free from the effects of corruption discussed in $\$ 2.1$. On inspection, it turned out that three of the four missing reflections had been rejected at the integration stage because they were saturated on the detector. These were the 200, 101 and 220 and their symmetry-related reflections. The 110 reflection and its symmetry mates had been excluded because it was too close to the beamstop. This is summarized in Table 3. Fig. 6 shows zoomed regions of $1^{\circ}$ 
Table 3

The status of all reflections between 284 and $125 \AA$.

A tick mark indicates those reflections and symmetry mates that were succesfully integrated and scaled. OL indicates reflection that were overloaded or saturated on the detector.

\begin{tabular}{lll}
\hline Miller indices & Resolution $(\AA)$ & Measured? \\
\hline 110 & 283.9 & Too close to beamstop \\
200 & 200.8 & OL \\
210 & 179.6 & $\sqrt{ }$ \\
101 & 160.0 & OL \\
111 & 148.7 & $\sqrt{ }$ \\
220 & 142.0 & OL \\
201 & 132.0 & $\sqrt{ }$ \\
310 & 127.0 & $\sqrt{ }$ \\
211 & 125.1 & $\sqrt{ }$ \\
\hline
\end{tabular}

images from the ribosome data where the 110 and 200 reflections are visible. The 110 reflection is just grazing the beamstop and has been rejected because it contains pixels which lie outside the defined active region of the detector (a $2.3 \mathrm{~mm}$ exclusion region was defined during integration with MOSFLM). It can be seen that although the 200 reflection has been rejected because it is saturated, it is nevertheless clearly resolved from its neighbouring reflections and from the beamstop. Similarly, the 101 and 220 reflections were well resolved but saturated. It is reasonable to assume that on remeasuring these reflections with a reduced exposure, integration would proceed normally.

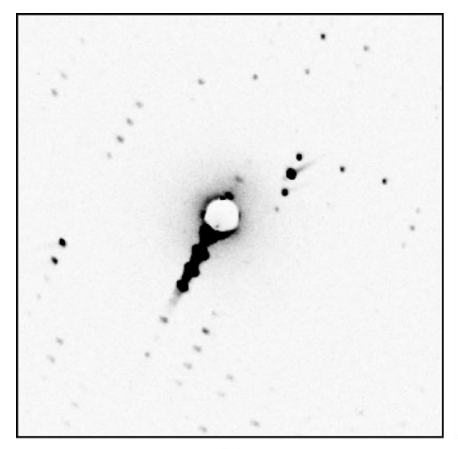

(a)

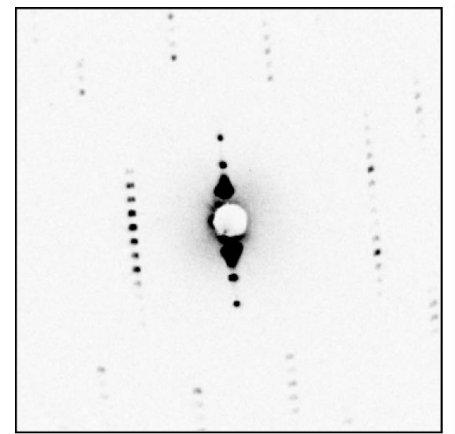

(c)

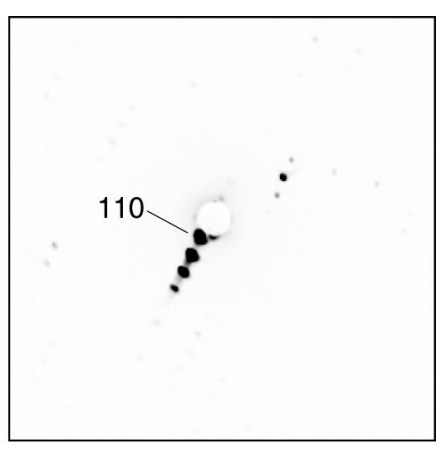

(b)

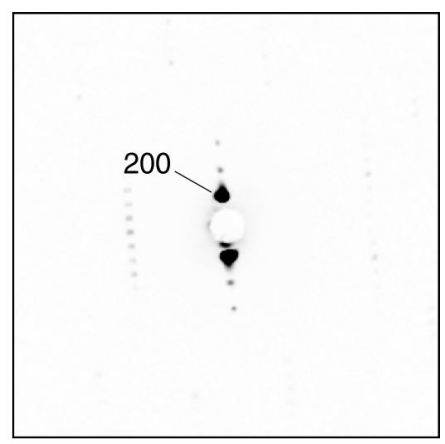

(d)
Figure 6

The central region of $1^{\circ}$ rotation images of very low angle diffraction from a tetragonal crystal of the $30 \mathrm{~S}$ ribosomal subunit. The images shown contain the 110 reflection at two contrast levels ( $a$ and $b$ ) and the 200 reflection $(c$ and $d$ ) also at two contrast levels.
Similar problems were encountered by Miller et al. (1999) during the in-house measurement of poliovirus crystal diffraction data. A small number of very low resolution reflections needed to be remeasured at much reduced exposure levels. These measurements were made however at the expense of reflections which were present in the longer exposure data set. This meant that there were no common reflections in the high- and low-exposure data sets which could be used to scale the data sets together. Scaling was performed solely on the basis of the recorded exposure times under the assumption that the beam intensity and diffracting volume were constant. In our study, the low-exposure pass through the data was not performed, but it is believed that similar scaling problems would have been encountered.

\section{Conclusions}

High-quality low-resolution data can be measured in-house with only minor modifications to existing protein crystallography hardware. The results of the contrast-variation experiment demonstrate that such effects can be measured in a straightforward way without the need for extraordinary apparatus or a synchrotron. Because the low-order reflections required in these methods are orders of magnitude stronger than the higher order reflections typically recorded by crystallographers, the measurements are particularly suited to in-house X-ray sources, which are in turn orders of magnitude weaker than synchrotron sources. The case of MASC, however, is an obvious exception because a tunable source of $\mathrm{X}$-rays is an absolute prerequisite.

The rapid measurement of very low resolution diffraction data from crystals of the $30 \mathrm{~S}$ ribosome has a number of implications. Firstly, the apparatus can allow convenient and quick screening of crystal diffraction at very low resolution which can be useful during the early stages of improvement of crystal-growth conditions where no high-angle diffraction is visible. More obviously, because many synchrotron beamlines are not permanently set up to measure very low resolution data, in-house measurements can potentially save on the use of synchrotron beam time.

We believe that the combination of routine in-house lowresolution data collection and existing contrast-variation dataanalysis techniques, as well as those that will appear in the next few years, will lead to a straightforward method for the determination of the protein envelope.

We are grateful to Venki Ramakrishnan and William Clemons for providing the $30 \mathrm{~S}$ ribosomal subunit crystal used during these studies. This work was partly supported by a Sponsored Research Agreement from Pfizer Central Research to GB.

\section{References}

Ban, N., Freeborn, B., Nissen, P., Penczek, P., Grassucci, R. A., Sweet, R., Frank, K., Moore, P. B. \& Steitz, T. A. (1998). Cell, 93, 11051115.

Bernal, J. \& Crowfoot, D. (1934). Nature (London), 133, 794-795. 
Bolduan, O. E. A. \& Bear, R. S. (1949). J. Appl. Phys. 20, 983-992. Böttcher, B., Wynne, S. A. \& Crowther, R. T. (1997). Nature (London), 386, 88-91.

Boyes-Watson, J., Davidson, E. \& Perutz, M. F. (1947). Proc. R. Soc. London Ser. A, 191, 83-132.

Bragg, W. L. \& Perutz, M. F. (1952). Acta Cryst. 5, 277-283.

Carter, C. W. Jr (1998). Direct Methods for Solving Macromolecular Structures, pp. 227-237. Netherlands: Kluwer Academic Publishers.

Carter, C. W. Jr, Crumley, K. V., Coleman, D. E., Hage, F. \& Bricogne, G. (1990). Acta Cryst. A46, 57-68.

Clemons, W. M. Jr, May, J. L. C., Wimberly, B. T., McCutcheon, J. P., Capel, M. S. \& Ramakrishnan, V. (1999). Nature (London), 400(6747), 833-840.

Collaborative Computational Project, Number 4 (1994). Acta Cryst. D50, 760-763.

Dokland, T., McKenna, R., Sherman, D. M., Bowman, B. R., Bean, W. F. \& Rossmann, M. G. (1998). Acta Cryst. D54, 878-890.

Forsythe, E. L., Nadarajah, A. \& Pusey, M. L. (1999). Acta Cryst. D55, 1005-1011.

Fourme, R., Shepard, W., Kahn, R., l'Hermite, G. \& de La Sierra, I. L. (1995). J. Synchrotron Rad. 2, 36-48.

Glykos, N. M., Holzenburg, A. \& Phillips, S. E. (1998). Acta Cryst. D54, 215-225.

Grimes, J. M., Jakana, J., Ghosh, M., Basak, A. K., Roy, P., Chiu, W., Stuart, D. I. \& Prasad, B. V. (1997). Structure, 5, 885-893.

Harms, J., Tocilj, A., Levin, I., Agmon, I., Stark, H., Kolln, I., van Heel, M., Cuff, M., Schlunzen, F., Bashan, A., Franceschi, F. \& Yonath, A. (1999). Structure Fold. Des. 7(8), 931-941.

Harrison, S. C. (1969). J. Mol. Biol. 42, 457-483.

Harrison, S. C. (1968). J. Appl. Cryst. 1, 84-90.
Harrison, S. C. \& Jack, A. (1975). J. Mol. Biol. 97, 173-191.

Huxley, H. E. (1953). Acta Cryst. 6, 457-465.

Jack, A., Harrison, S. C. \& Crowther, R. A. (1975). J. Mol. Biol. 97, 163-172.

Jiang, J. S. \& Brunger, A. T. (1994). J. Mol. Biol. 243(1), 100-115.

Leslie, A. G. W. (1992). Jnt CCP4/ESF-EAMCB Newslett. Protein Crystallogr. 26.

Lunin, V., Lunina, N., Petrova, T., Urzhumtsev, A. \& Podjarny, A. (1998). Acta Cryst. D54(5), 726-734.

Miller, S. T., Genova, J. D. \& Hogle, J. M. (1999). J. Appl. Cryst. 32, 1183-1185.

Perutz, M. F. \& Rogers, G. L. (1946). J. Sci. Instrum. 23(9), 217.

Phillips, S. E. V. (1980). J. Mol. Biol. 142(4), 531-554.

Prasad, B. V., Hardy, M. E., Dokland, T., Bella, J., Rossmann, M. G. \& Estes, M. K. (1999). Science, 286, 287-290.

Ramin, M., Shepard, W., Fourme, R. \& Kahn, R. (1999). Acta Cryst. D55, 157-167.

Rayment, I., Baker, T. S., Caspar, D. L. \& Murakami, W. T. (1982). Nature (London), 295(5845), 110-115.

Simpson, A. A., Chipman, P. R., Baker, T. S., Tijssen, P. \& Rossmann, M. G. (1998). Structure, 6(11), 1355-1367.

Subbiah, S. (1991). Science, 252, 128-133.

Svergun, D. I., Richard, S., Koch, M. H., Sayers, Z., Kuprin, S. \& Zaccai, G. (1998). Proc. Natl Acad. Sci. USA, 95(5), 2267-2272.

Tsuruta, H., Reddy, V. S., Wikoff, W. R. \& Johnson, J. E. (1998). J. Mol. Biol. 284(5), 1439-1452.

Wang, J., Hartling, J. A. \& Flanagan, J. (1997). Cell, 91, 447-456.

Wynne, S. A., Crowther, R. T. \& Leslie, A. G. W. (1999). Mol. Cell, 3, 771-780.

Yu, B. \& Caspar, D. L. D. (1998). Biophys. J. 74, 616-622. 